\title{
ACCUMULATION RATE OF BOUND STATES OF DIPOLES GENERATED BY POINT CHARGES IN STRAINED GRAPHENE
}

\author{
FLORIAN DORSCH*
}

\begin{abstract}
We consider strained graphene, modelled by the two-dimensional massive Dirac operator, with potentials corresponding to charge distributions with vanishing total charge, non-vanishing dipole moment and finitely many point charges of subcritical coupling constants located in the graphene sheet. We show that the bound state energies accumulate exponentially fast at the edges of the spectral gap by determining the leading order of the accumulation rate.
\end{abstract}

\section{INTRODUCTION}

Electrons close to the Fermi level in strained graphene can be described by the twodimensional massive Dirac operator [17]. De Martino et al [3] predicted the existence of infinitely many bound states of the two-dimensional massive Dirac operator with a dipole potential and that these bound states accumulate with an exponential rate at the edges of the spectral gap. Shortly after, Cuenin and Siedentop [1] proved the former statement, whereas the latter one has so far only been proven under the assumption that no point charges are located directly in the graphene sheet (Rademacher and Siedentop [10]). The purpose of the present article is to extend the result in [10] to the case of potentials with finitely many such Coulomb singularities with subcritical coupling constants.

The operator of interest acts in $L^{2}\left(\mathbb{R}^{2}, \mathbb{C}^{2}\right)$ and is formally given by the expression

$$
\begin{aligned}
F & =-i \sigma \cdot \nabla+m \sigma_{3}+V, \\
V & =V_{\text {sing }}+V_{\text {reg }}, \\
V_{\text {sing }} & =\sum_{n=1}^{N} v_{n}\left|\cdot-x_{n}\right|^{-1}
\end{aligned}
$$

with $\boldsymbol{\sigma}=\left(\sigma_{1}, \sigma_{2}\right)$, where $\left\{\sigma_{k}\right\}_{k=1}^{3}$ are the standard Pauli matrices, $m \in \mathbb{R}^{+}:=(0, \infty)$ is a strictly positive mass and $V$ is the (real-valued) potential associated to the charge distribution given by a finite, signed Borel measure $\rho$ in $\mathbb{R}^{3}$ via

$$
V: \mathbb{R}^{2} \rightarrow \mathbb{R}, \quad x \mapsto \int_{\mathbb{R}^{3}} \frac{\mathrm{d} \rho(y)}{|(x, 0)-y|},
$$

where the charge distribution is, accordingly, split into a singular and regular part, viz.,

$$
\begin{aligned}
\rho & =\rho_{\text {sing }}+\rho_{\text {reg }}, \\
\rho_{\text {sing }} & =\sum_{n=1}^{N} v_{n} \delta\left(\cdot-\left(x_{n}, 0\right)\right),
\end{aligned}
$$

where the positions $\left\{x_{n}\right\}_{n=1}^{N} \subset \mathbb{R}^{2}$ of the charges of subcritical coupling constants $\left\{v_{n}\right\}_{n=1}^{N} \subset(-1 / 2,1 / 2) \backslash\{0\}$ are mutually distinct, i.e., $y_{k} \neq y_{j}$ whenever $k \neq j$.

\footnotetext{
*Mathematisches Institut, LMU München, Theresienstr. 39, 80333 Munich, Germany florian.dorsch@gmx. org
} 
The assumptions on $\rho_{\text {reg }}$ will be made in Thm. 7.

We denote the dipole moment corresponding to $\rho$ by

$$
\mathfrak{d}:=\int_{\mathbb{R}^{3}}\left(y_{1}, y_{2}\right) \mathrm{d} \rho(y) \in \mathbb{R}^{2}
$$

and the radius of a sufficiently large ball around the Coulomb singularities by

$$
\gamma:=2 \max _{n \in\{1, \ldots, N\}}\left\{\left|x_{n}\right|\right\}
$$

In order to obtain a physically sensible self-adjoint realization of $F$, we will recall two basic facts proven in [1].

Proposition 1 (distinguished self-adjoint extension (cf. [1], Thm. 1, Rem. 1)). The operator $-i \sigma \cdot \nabla+m \sigma_{3}+V_{\text {sing }}$ defined on $C_{0}^{\infty}\left(\mathbb{R}^{2} \backslash\left\{x_{n}\right\}_{n=1}^{N}, \mathbb{C}^{2}\right)$ has a unique self-adjoint extension $\tilde{D}$ satisfying $\mathscr{D}(\tilde{D}) \subset \mathrm{H}^{1 / 2}\left(\mathbb{R}^{2}, \mathbb{C}^{2}\right)$.

Proposition 2 (energy gap ([1], Prop. 1)). The essential spectrum of $\tilde{D}$ is given by $\sigma_{\mathrm{ess}}(\tilde{D})=\mathbb{R} \backslash(-m, m)$.

To formulate our main result, we will need the following definitions.

Definition 3 (rescaled Mathieu operator). For $p \in \mathbb{R}^{+}$we define the rescaled Mathieu operator with periodic boundary conditions on the domain $\mathscr{D}\left(M_{p}\right)=\mathrm{H}^{2}\left(\mathbb{S}^{1}\right)$ as

$$
M_{p} \psi:=\left(-\partial^{2}-p \cos (\cdot)\right) \psi .
$$

Definition 4. For a self-adjoint operator $A$ and a Borel set $I \subset \mathbb{R} \backslash \sigma_{\text {ess }}(A)$ we define the number of eigenvalues (counting multiplicity) by

$$
\mathscr{N}_{I}(A):=\operatorname{rank}\left(\chi_{I}(A)\right),
$$

where $\chi$ denotes the indicator function.

Definition 5. We denote a ball of radius $a \in \mathbb{R}_{0}^{+} \cup\{\infty\}$ by $B_{a}:=\left\{x \in \mathbb{R}^{2}:|x|<a\right\}$.

Definition 6. We introduce the effective rest potential $R$, which is obtained from the potential $V$ by subtracting the short range part of $V_{\text {sing }}$ and the long range part of the pure point dipole, i.e.,

$$
R: \mathbb{R}^{2} \rightarrow \mathbb{R}, \quad x \mapsto V_{\text {reg }}(x)+\left[V_{\text {sing }}(x)-\frac{\langle\mathfrak{d}, x\rangle_{\mathbb{R}^{2}}}{|x|^{3}}\right] \chi_{\mathbb{R}^{2} \backslash B_{\gamma}}(x) .
$$

The Kato-Rellich theorem implies that

$$
D:=\tilde{D}+V_{\text {reg }}
$$

is self-adjoint if the regular part of the potential $V_{\text {reg }}$ is relatively $\tilde{D}$-bounded with relative bound $n_{\tilde{D}}\left(V_{\text {reg }}\right)<1$. 
Theorem 7 (exponential accumulation rate). Let $\tilde{D}$ be the distinguished self-adjoint extension of $-i \boldsymbol{\sigma} \cdot \nabla+m \sigma_{3}+V_{\text {sing }}$ defined on $\mathrm{C}_{0}^{\infty}\left(\mathbb{R}^{2} \backslash\left\{x_{n}\right\}_{n=1}^{N}, \mathbb{C}^{2}\right)$ (see Prop. (1). Assume that the following hypotheses hold:

1. The regular part of the potential $V_{\text {reg }}$ is relatively $\tilde{D}$-bounded with relative bound $n_{\tilde{D}}\left(V_{\text {reg }}\right)<1$.

2. The square of the regular part of the potential $\left(V_{\mathrm{reg}}\right)^{2}$ is relatively compact w.r.t. the Laplacian $-\Delta_{\mathbb{R}^{2}}$ defined on $\mathrm{H}^{2}\left(\mathbb{R}^{2}\right)$.

3. The dipole moment $\mathfrak{d}$ (see (4)) is non-zero: $\mathfrak{d} \neq 0$.

4. There are neighborhoods of the positions $\left\{x_{n}\right\}_{n=1}^{N}$ of the point charges in which the regular part of the potential $V_{\text {reg }}$ is bounded.

5. The effective rest potential $R$ (see Def. 6) fulfills the following integrability conditions:

(a) $R, R^{2} \in \mathrm{L}^{1}\left(\mathbb{R}^{2} ; \log (2+|x|) \mathrm{d} x\right)$.

(b) $|R|_{*},\left(R^{2}\right)_{*} \in \mathrm{L}^{1}\left(\mathbb{R}^{+} ; \log _{+}\left(r^{-1}\right) \mathrm{d} r\right)$.

Here, $(\cdot)_{*}$ denotes the (non-increasing) spherical rearrangement (see [15] ).

Then, $D$, defined by (9), satisfies

$$
\lim _{E \nearrow m} \frac{\mathscr{N}_{(-E, E)}(D)}{|\log (m-E)|}=\frac{1}{\pi} \operatorname{tr}\left(\sqrt{\left(M_{2 m|\mathfrak{d}|}\right)_{-}}\right),
$$

where $(\cdot)_{-}:=-\min \{\cdot, 0\}$ denotes the negative part.

Remark 8. Hypothesis 5. (a) in Thm. 7)ensures that the total charge vanishes.

Remark 9. The expression on the right of (10) is always strictly positive, since the lowest eigenvalue of $M_{p}$ is negative for all $p \in \mathbb{R}^{+}$(see [6], 3.25, diagram).

\section{The Two-Dimensional Massless Dirac Operator With SubCritical Coulomb Potential}

Let $v \in(-1 / 2,1 / 2) \backslash\{0\}$. Since the differential expression of the two-dimensional massless Dirac operator with Coulomb potential $-i \boldsymbol{\sigma} \cdot \nabla+v|\cdot|^{-1}$ acting in $\mathrm{L}^{2}\left(B_{a}, \mathbb{C}^{2}\right)$, where $a \in \mathbb{R}^{+} \cup\{\infty\}$, commutes with the total angular momentum $-i \partial_{\varphi}+\frac{1}{2} \sigma_{3}$, it can be decomposed by a unitary map $\mathscr{U}_{a}: \mathrm{L}^{2}\left(B_{a}, \mathbb{C}^{2}\right) \rightarrow \bigoplus_{\kappa \in \mathbb{Z}+1 / 2} \mathrm{~L}^{2}\left((0, a), \mathbb{C}^{2}\right)$ into

$$
\bigoplus_{\kappa \in \mathbb{Z}+1 / 2} \mathbf{d}_{\kappa}^{v}, \quad \text { where } \quad \mathbf{d}_{\kappa}^{v}:=\left(\begin{array}{cc}
\frac{v}{r} & -\partial_{r}-\frac{\kappa}{r} \\
\partial_{r}-\frac{\kappa}{r} & \frac{v}{r}
\end{array}\right)
$$

(see [16], Section 7.3.3). We define for $\kappa \in \mathbb{Z}+1 / 2$ the operator

$$
d_{\kappa, a}^{v}: \mathrm{C}_{0}^{\infty}\left((0, a), \mathbb{C}^{2}\right) \rightarrow \mathrm{L}^{2}\left((0, a), \mathbb{C}^{2}\right), \quad \psi \mapsto \mathbf{d}_{\kappa}^{v} \psi
$$




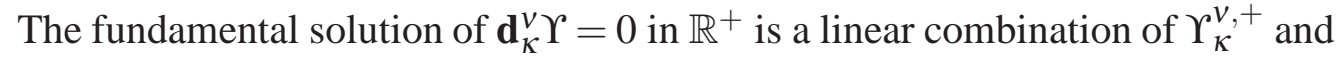
$\Upsilon_{\kappa}^{v,-}$, where

$$
\Upsilon_{\kappa}^{v, \pm}(r)=\left(\begin{array}{c}
-v \\
\pm \sqrt{\kappa^{2}-v^{2}}-\kappa
\end{array}\right) r^{ \pm \sqrt{\kappa^{2}-v^{2}}}
$$

and hence $\mathbf{d}_{\kappa}^{v}$ is in the limit circle case at $\vartheta \in \mathbb{R}^{+}$and in the limit point case at $\infty$ and, moreover, it is in the limit circle case at 0 if and only if $\kappa= \pm 1 / 2$ [7]. Thus, the deficiency indices of $d_{\kappa, \infty}^{v}$ are $(1,1)$ in case of $\kappa= \pm 1 / 2$ and $(0,0)$, otherwise [7],[19]; the deficiency indices of $d_{\kappa, \vartheta}^{v}$ are $(2,2)$ in case of $\kappa= \pm 1 / 2$ and $(1,1)$, otherwise [19].

We define

$$
D_{a}^{v}: \mathrm{C}_{0}^{\infty}\left(B_{a} \backslash\{0\}, \mathbb{C}^{2}\right) \rightarrow \mathrm{L}^{2}\left(B_{a}, \mathbb{C}^{2}\right), \quad \psi \mapsto\left(-i \boldsymbol{\sigma} \cdot \nabla+v|\cdot|^{-1}\right) \psi
$$

and denote the distinguished self-adjoint extension (see Prop. 1) of $D_{\infty}^{v}$ by $H_{\infty}^{v}$.

Lemma 10. For all $\vartheta \in \mathbb{R}^{+}$there exists a self-adjoint extension $H_{\vartheta}^{v}$ of $D_{\vartheta}^{v}$ with discrete spectrum.

Proof. It suffices to find self-adjoint extensions $\left\{\hat{d}_{\kappa, \vartheta}^{v}\right\}_{\kappa \in \mathbb{Z}+1 / 2}$ of $\left\{d_{\kappa, \vartheta}^{v}\right\}_{\kappa \in \mathbb{Z}+1 / 2}$ with compact resolvents and the property that

$$
\left\|\left(\hat{d}_{\kappa, \vartheta}^{v}+i \mathbb{I}\right)^{-1}\right\| \longrightarrow 0 \quad \text { as } \quad \kappa \rightarrow \pm \infty .
$$

In case of $\kappa= \pm 1 / 2$, the resolvent of any self-adjoint extension of $d_{\kappa, \vartheta}^{\nu}$ is a HilbertSchmidt operator, since $\mathbf{d}_{\kappa}^{v}$ is in the limit circle case both at 0 and at $\vartheta$ (see [19], Prop. 1.6). Now, let $\kappa \neq \pm 1 / 2$. A self-adjoint extension of $d_{\kappa, \vartheta}^{v}$ is given by

$$
\begin{array}{r}
\hat{d}_{\kappa, \vartheta}^{v}: \mathscr{D}\left(\hat{d}_{\kappa, \vartheta}^{v}\right) \rightarrow \mathrm{L}^{2}\left((0, \vartheta), \mathbb{C}^{2}\right), \quad \psi \mapsto \mathbf{d}_{\kappa}^{v} \psi, \\
\text { where } \quad \mathscr{D}\left(\hat{d}_{\kappa, \vartheta}^{v}\right):=\left\{\phi \equiv\left(\phi_{1}, \phi_{2}\right) \in \mathrm{L}^{2}\left((0, \vartheta), \mathbb{C}^{2}\right) \cap \mathrm{AC}_{\mathrm{loc}}\left((0, \vartheta), \mathbb{C}^{2}\right):\right. \\
\left.\mathbf{d}_{\kappa}^{v} \phi \in \mathrm{L}^{2}\left((0, \vartheta), \mathbb{C}^{2}\right), \phi_{1}(\vartheta)=\phi_{2}(\vartheta)\right\} .
\end{array}
$$

Indeed, observing that the conditions $\phi_{1}(\vartheta)=\phi_{2}(\vartheta)$ and $\left\langle i \sigma_{2} \phi(\vartheta), \Upsilon_{\kappa}^{v}(\vartheta)\right\rangle_{\mathbb{C}^{2}}=0$, where

$$
\Upsilon_{\kappa}^{v}:=\left(\sqrt{\kappa^{2}-v^{2}}-v+\kappa\right) \Upsilon_{\kappa}^{v,+}+\vartheta^{2 \sqrt{\kappa^{2}-v^{2}}}\left(\sqrt{\kappa^{2}-v^{2}}+v-\kappa\right) \Upsilon_{\kappa}^{v,-},
$$

are equivalent, this follows from Prop. 1.5 in [19], since $\Upsilon_{K}^{\nu}$ solves $\mathbf{d}_{\kappa}^{v} \Upsilon=0$.

There are two functions $\Omega_{\kappa}^{v, \pm}: \mathbb{R}^{+} \rightarrow \mathbb{C}^{2}$ with

$$
\Omega_{\kappa}^{v,+}(r)=r^{\sqrt{\kappa^{2}-v^{2}}}\left(\begin{array}{c}
\kappa+\sqrt{\kappa^{2}-v^{2}} \\
v
\end{array}\right)+\mathscr{O}\left(r^{1+\sqrt{\kappa^{2}-v^{2}}}\right)
$$

and

$$
\Omega_{\kappa}^{v,-}(r)=r^{-\sqrt{\kappa^{2}-v^{2}}}\left(\begin{array}{c}
v \\
\kappa+\sqrt{\kappa^{2}-v^{2}}
\end{array}\right)+\mathscr{O}\left(r^{1-\sqrt{\kappa^{2}-v^{2}}}\right)
$$


as $r \rightarrow 0$ generating the fundamental solution of $\left(\mathbf{d}_{\kappa}^{v}+i\right) \Omega=0$ in $\mathbb{R}^{+}$(see [8], Lem. 8). For any $c \in(0, \vartheta)$, the restriction of $\Omega_{\mathcal{K}}^{v,-}$ to $(0, c)$ is not contained in $\mathrm{L}^{2}\left((0, c), \mathbb{R}^{2}\right)$. Therefore, the integral kernel of $\left(\hat{d}_{\kappa, \vartheta}^{v}+i \mathbb{I}\right)^{-1}$ is given by

$$
G_{\kappa, \vartheta}^{v}:(0, \vartheta)^{2} \rightarrow \mathbb{C}^{2 \times 2}, \quad(x, y) \mapsto \text { const. } \begin{cases}\Omega_{\kappa}^{v,+}(x)\left(\Omega_{\kappa, \vartheta}^{v}(y)\right)^{\top}, & x<y, \\ \Omega_{\kappa, \vartheta}^{v}(x)\left(\Omega_{\kappa}^{v,+}(y)\right)^{\top}, & x \geq y,\end{cases}
$$

where $\Omega_{\kappa, \vartheta}^{v}$ is the solution of $\left(\mathbf{d}_{\kappa}^{v}+i\right) \Omega=0$ satisfying $\left\langle i \sigma_{2} \Omega_{\kappa, \vartheta}^{v}(\vartheta), \Upsilon_{\kappa}^{v}(\vartheta)\right\rangle_{\mathbb{C}^{2}}=0$ (see [19], Prop. 1.6). It follows from (18), (19) and the continuity of $\Omega_{\kappa}^{v, \pm}$ on $(0, \vartheta]$ that the components of $\Omega_{\kappa}^{v,+}(r)$ and $\Omega_{\kappa, \vartheta}^{v}(r)$ are bounded on $r \in(0, \vartheta)$ by $C r^{\sqrt{\kappa^{2}-v^{2}}}$ and $C r^{-\sqrt{\kappa^{2}-v^{2}}}$ for some $C \in \mathbb{R}^{+}$, respectively. Thus, $G_{\kappa, \vartheta}^{v}$ lies in $\mathrm{L}^{2}\left((0, \vartheta)^{2}, \mathbb{C}^{2 \times 2}\right)$ and hence $\left(\hat{d}_{\kappa, \vartheta}^{v}+i \mathbb{I}\right)^{-1}$ is a Hilbert-Schmidt operator. A core for $\hat{d}_{\kappa, \vartheta}^{v}$ is given by $\mathfrak{C}^{\vartheta}:=\left\{\left(\phi_{1}, \phi_{2}\right) \in \mathrm{C}_{0}^{\infty}\left((0, \vartheta], \mathbb{C}^{2}\right): \phi_{1}(\vartheta)=\phi_{2}(\vartheta)\right\}$, since the closure of the restriction of $\hat{d}_{\kappa, \vartheta}^{v}$ to $\mathfrak{C}^{\vartheta}$ is a strict extension of $\overline{d_{\kappa, \vartheta}^{v}}$. Indeed, for instance, $f:=(1,1) \lim _{x \downarrow \max \{\cdot, \vartheta / 2\}} \exp [2 /(\vartheta-2 x)] \in \mathfrak{C}^{\vartheta} \backslash \mathscr{D}\left(\overline{d_{\kappa, \vartheta}^{v}}\right)$, as $\left\langle i \sigma_{2} f(\vartheta), \Upsilon_{\kappa}^{v,+}(\vartheta)\right\rangle_{\mathbb{C}^{2}} \neq 0$ (see [19], Prop. 1.5). Now, let $\psi \equiv\left(\psi_{1}, \psi_{2}\right) \in \mathfrak{C}^{\vartheta}$. Then, $2 \vartheta\left\|\hat{d}_{\kappa, \vartheta}^{v} \psi\right\| \geq|\kappa|\|\psi\|$ holds for large $|\kappa|$, hence (15) is satisfied. Indeed,

$$
\begin{aligned}
& \left\|\hat{d}_{\kappa, \vartheta}^{v} \psi\right\|^{2}=\overbrace{\left\|\hat{d}_{3 / 2, \vartheta}^{v} \psi\right\|^{2}}^{\geq 0}+\int_{0}^{\vartheta} \mathrm{d} r\left(\frac{|2 \kappa-3|^{2}+6(2 \kappa-3)}{4 r^{2}}|\psi(r)|^{2}-\right. \\
& \underbrace{-\frac{2(2 \kappa-3) v}{r^{2}} \Re\left[\overline{\psi_{1}(r)} \psi_{2}(r)\right]}_{\geq-\frac{|2 \kappa-3||v|}{r^{2}}|\psi(r)|^{2}}-\frac{2 \kappa-3}{2 r} \partial_{r}\left[\left|\psi_{1}(r)\right|^{2}-\left|\psi_{2}(r)\right|^{2}\right]) \\
& =: S_{\kappa}^{v}[\psi] \\
& \geq \overbrace{\frac{1}{4}\left[|2 \kappa-3|^{2}+6(2 \kappa-3)-4|2 \kappa-3|\left(|v|+\frac{1}{2}\right)\right] \int_{0}^{\vartheta} \mathrm{d} r\left|\frac{\psi(r)}{r}\right|^{2}}+ \\
& +\frac{1}{2} \underbrace{\left(|2 \kappa-3| \int_{0}^{\vartheta} \mathrm{d} r\left|\frac{\psi(r)}{r}\right|^{2}-(2 \kappa-3) \int_{0}^{\vartheta} \mathrm{d} r \frac{1}{r} \partial_{r}\left[\left|\psi_{1}(r)\right|^{2}-\left|\psi_{2}(r)\right|^{2}\right]\right)}_{=: T_{\kappa}[\psi]} .
\end{aligned}
$$

Exploiting the boundary condition at $r=\vartheta$, integration by parts yields

$$
\int_{0}^{\vartheta} \mathrm{d} r \frac{1}{r} \partial_{r}\left[\left|\psi_{1}(r)\right|^{2}-\left|\psi_{2}(r)\right|^{2}\right]=\int_{0}^{\vartheta} \mathrm{d} r \frac{1}{r^{2}}\left[\left|\psi_{1}(r)\right|^{2}-\left|\psi_{2}(r)\right|^{2}\right]
$$

and, therefore, $T_{\kappa}[\psi]$ is nonnegative. But for large $|\kappa|$ it holds that

$$
4 S_{\kappa}^{\nu}[\psi] \geq \kappa^{2} \int_{0}^{\vartheta} \mathrm{d} r\left|\frac{\psi(r)}{r}\right|^{2} \geq \frac{\kappa^{2}}{\vartheta^{2}}\|\psi\|^{2}
$$


Lemma 11. For all $\vartheta \in \mathbb{R}^{+}$there exist $f_{\vartheta}, g_{\vartheta} \in \mathrm{H}^{1 / 2}\left(B_{\vartheta}, \mathbb{C}^{2}\right)$ so that the restriction of $H_{\infty}^{v}$ to $C_{0}^{\infty}\left(\mathbb{R}^{2} \backslash\{0\}, \mathbb{C}^{2}\right) \dot{+} \operatorname{span}\left\{f_{\vartheta}, g_{\vartheta}\right\}$ is essentially self-adjoint.

Proof. For $\kappa \neq \pm 1 / 2$ the operator $d_{\kappa, \infty}^{\nu}$ is essentially self-adjoint (see above). Now, let $\kappa= \pm 1 / 2$. We pick $\varsigma \in \mathrm{C}_{0}^{\infty}([0, \vartheta))$ such that $\varsigma(2 w)=1 \quad \forall w \leq \vartheta$. Then, it holds that $\varsigma \Upsilon_{\kappa}^{v, \pm} \notin \mathscr{D}\left(\overline{d_{\kappa, \infty}^{v}}\right)$, since $\lim _{r \rightarrow 0}\left\langle i \sigma_{2} \varsigma(r) \Upsilon_{\kappa}^{v, \pm}(r), \Upsilon_{\kappa}^{v, \mp}(r)\right\rangle_{\mathbb{C}^{2}} \neq 0$ (see [19], Prop. 1.5), and therefore

$$
\mathscr{D}\left(\left(d_{\kappa, \infty}^{v}\right)^{*}\right)=\mathscr{D}\left(\overline{d_{\kappa, \infty}^{v}}\right) \dot{+} \operatorname{span}\left\{\varsigma \Upsilon_{\kappa}^{v,+}, \varsigma \Upsilon_{\kappa}^{\nu,-}\right\} .
$$

Thus, any self-adjoint extension of $d_{\kappa, \infty}^{v}$ is obtained as the closure of the restriction of $\left(d_{\kappa, \infty}^{v}\right)^{*}$ to $C_{0}^{\infty}\left(\mathbb{R}^{+}, \mathbb{C}^{2}\right) \dot{+} \operatorname{span}\left\{\varsigma\left(\alpha \Upsilon_{\kappa}^{v,+}+\beta \Upsilon_{\kappa}^{\nu,-}\right)\right\}$ with $(\alpha, \beta) \in \mathbb{C}^{2} \backslash\{0\}$; the distinguished one is obtained by setting $(\alpha, \beta)=(1,0)$. Indeed, one may verify that $\mathscr{U}_{\infty}^{*} \mathfrak{P}_{\kappa}\left[\varsigma\left(\alpha \Upsilon_{\kappa}^{v,+}+\beta \Upsilon_{\kappa}^{v,-}\right)\right] \notin \mathrm{L}^{2}\left(\mathbb{R}^{2}, \mathbb{C}^{2} ;|x|^{-1} \mathrm{~d} x\right)$ for all $(\alpha, \beta) \in \mathbb{C}^{2} \backslash \operatorname{span}\{(1,0)\}$, where $\mathfrak{P}_{\kappa}: \mathrm{L}^{2}\left(\mathbb{R}^{+}, \mathbb{C}^{2}\right) \rightarrow \underset{\lambda \in \mathbb{Z}+1 / 2}{\bigoplus} \mathrm{L}^{2}\left(\mathbb{R}^{+}, \mathbb{C}^{2}\right), f \mapsto \underset{\lambda \in \mathbb{Z}+1 / 2}{\bigoplus} \delta_{\lambda, \kappa} f$, where $\delta_{\text {. }}$, is the

Kronecker symbol. But $\mathrm{H}^{1 / 2}\left(\mathbb{R}^{2}\right) \subset \mathrm{L}^{2}\left(\mathbb{R}^{2} ;\left(1+|x|^{-1}\right) \mathrm{d} x\right)$ (cf. [9], Rem. 15).

\section{Proof of the Exponential Accumulation Rate}

It follows from Hypothesis 4 in Thm. 7 that we can choose

$$
\varepsilon \in\left(0, \min \left\{\left|x_{j}-x_{k}\right| / 3: j, k \in\{1, \ldots, N\}, j \neq k\right\}\right)
$$

such that $V_{\text {reg }}$ is bounded on $\left(B_{\varepsilon}+x_{n}\right)$ for all $n \in\{1, \ldots, N\}$.

In order to localize the Coulomb singularities, we pick a partition of unity $\left\{U_{n}\right\}_{n=0}^{N}$ with the following properties:

- $\left\{U_{n}\right\}_{n=0}^{N} \subset C^{\infty}\left(\mathbb{R}^{2},[0,1]\right)$,

- $\sum_{n=0}^{N}\left(U_{n}\right)^{2}=1$

- $\operatorname{supp}\left(U_{n}\right) \in B_{\varepsilon}+x_{n}$ for all $n \in\{1, \ldots, N\}$,

- $\operatorname{supp}\left(U_{0}\right) \subset \mathbb{R}^{2} \backslash \bigcup_{n=1}^{N}\left(\overline{B_{\varepsilon / 2}}+x_{n}\right)$.

Thanks to the spectral theorem, we can devote ourselves to the study of the asymptotic behavior of the negative eigenvalues of $D^{2}-m^{2} \mathbb{I}$ using the Min-Max principle (see [13], Thm. 12.1). The Min-Max values of a lower semi-bounded selfadjoint operator $A$ are denoted by $\mu_{(\cdot)}(A)$ (see [13], formula (12.2)). The following lemma guarantees that we can restrict the minimization to $\mathfrak{C}:=\mathrm{C}_{0}^{\infty}\left(\mathbb{R}^{2} \backslash\left\{x_{n}\right\}_{n=1}^{N}, \mathbb{C}^{2}\right)$ when investigating the asymptotic behavior of the eigenvalues.

Lemma 12. The defect number of the restriction of $D$ to $\mathfrak{C}$ is bounded above by $2 N$.

Proof. It follows from Lem. 11 that for all $n \in\{0, \ldots, N\}$ the restriction of $U_{n} \tilde{D} U_{n}$ to $\tilde{\mathfrak{C}}:=\mathfrak{C} \dot{+} \operatorname{span}\left(\left\{f_{\varepsilon}\left(\cdot-x_{j}\right), g_{\varepsilon}\left(\cdot-x_{j}\right)\right\}_{j=1}^{N}\right)$ is essentially self-adjoint. Hence, given $\psi \in \mathscr{D}(\tilde{D})$ and $n \in\{0, \ldots, N\}$, we can choose a sequence $\left\{\psi_{k}^{(n)}\right\}_{k \in \mathbb{N}} \subset \tilde{\mathfrak{C}}$ such that $\psi_{k}^{(n)} \rightarrow \psi$ as $k \rightarrow \infty$ w.r.t. $\|\cdot\|_{U_{n} \tilde{D} U_{n}}$. For $k \in \mathbb{N}$ we define $\phi_{k}:=\sum_{n=0}^{N}\left(U_{n}\right)^{2} \psi_{k}^{(n)}$ and observe that $\left\{\phi_{k}\right\}_{k \in \mathbb{N}} \subset \tilde{\mathfrak{C}}$ and $\phi_{k} \rightarrow \psi$ as $k \rightarrow \infty$ w.r.t. $\|\cdot\|_{\tilde{D}}$. Thus, $\tilde{\mathfrak{C}}$ is a core for $\tilde{D}$ and - by the Kato-Rellich theorem [12] and Hypothesis11in Thm.7- also for $D$. 
Following Rademacher and Siedentop [10], we will deal with Schrödinger operators with relatively compact perturbations of $-\Delta_{\mathbb{R}^{2}}: \mathrm{H}^{2}\left(\mathbb{R}^{2}\right) \rightarrow \mathrm{L}^{2}\left(\mathbb{R}^{2}\right), \psi \mapsto-\Delta \psi$ whose eigenvalues bound those of $D^{2}-m^{2} \mathbb{I}$. It follows by the inequality of Seiler and Simon (see [14], Lem. 2.1) that $W_{2}\left(-\Delta_{\mathbb{R}^{2}}+\mathbb{I}\right)^{-1}$ is a Hilbert-Schmidt operator for all $W_{2} \in \mathrm{L}^{2}\left(\mathbb{R}^{2}\right)$. As the operator norm limit preserves compactness, any potential that lies in

$$
\begin{array}{r}
\mathrm{L}_{\infty}^{2}\left(\mathbb{R}^{2}\right):=\left\{W: \mathbb{R}^{2} \rightarrow \mathbb{C}: \forall \varepsilon \in \mathbb{R}^{+} \exists\left(W_{2}, W_{\infty}\right) \in \mathrm{L}^{2}\left(\mathbb{R}^{2}\right) \times \mathrm{L}^{\infty}\left(\mathbb{R}^{2}\right)\right. \\
\text { such that } \left.W=W_{2}+W_{\infty} \text { and }\left\|W_{\infty}\right\|_{\infty}<\varepsilon\right\}
\end{array}
$$

is relatively compact w.r.t. $-\Delta_{\mathbb{R}^{2}}$. For any such $\left(-\Delta_{\mathbb{R}^{2}}\right)$-compact potential $W$, the operator $-\Delta_{\mathbb{R}^{2}}+W$ is bounded from below and its restriction to $C_{0}^{\infty}\left(\mathbb{R}^{2}\right)$ is essentially self-adjoint (see [18], Section 17.2).

Hypothesis 5. (a) in Thm. 7 implies that $V_{\text {reg }} \in \mathrm{L}_{\infty}^{2}\left(\mathbb{R}^{2}\right)$. Therefore, $V \chi_{\operatorname{supp}\left(U_{0}\right)}$ and $W:=V_{\text {sing }}\left[V_{\text {sing }}+2 V_{\text {reg }}\right] \chi_{\text {supp }\left(U_{0}\right)}$ lie in $\mathrm{L}_{\infty}^{2}\left(\mathbb{R}^{2}\right)$, since $V_{\text {sing }} \chi_{\text {supp }\left(U_{0}\right)}$ is bounded and vanishes at infinity. Furthermore, $\left(V_{\text {reg }}\right)^{2}$ is $\left(-\Delta_{\mathbb{R}^{2}}\right)$-compact (see Hypothesis 2 in Thm. (7). Thus, both $V \chi_{\operatorname{supp}\left(U_{0}\right)}$ and $V^{2} \chi_{\operatorname{supp}\left(U_{0}\right)}=\left(V_{\text {reg }}\right)^{2} \chi_{\operatorname{supp}\left(U_{0}\right)}+W$ are relatively compact perturbations of $-\Delta_{\mathbb{R}^{2}}$ and the operator

$$
\mathfrak{V}_{ \pm}^{\zeta}:=-\Delta_{\mathbb{R}^{2}}+\left( \pm 2 m V \chi_{\operatorname{supp}\left(U_{0}\right)}+(1-1 / \zeta) V^{2} \chi_{\operatorname{supp}\left(U_{0}\right)}-\sum_{n=0}^{N}\left|\nabla U_{n}\right|^{2}\right) /(1-\zeta)
$$

is self-adjoint and bounded from below for all $\zeta \in(0,1)$. Moreover, $\mathrm{C}_{0}^{\infty}\left(\mathbb{R}^{2}\right)$ is a core for $\mathfrak{V}_{ \pm}^{\zeta}$. Obviously, $V \chi_{\mathbb{R}^{2} \backslash B_{\gamma}} \in \mathrm{L}_{\infty}^{2}\left(\mathbb{R}^{2}\right)$ and, therefore, the operator

$$
\tilde{\mathfrak{W}}_{ \pm}^{\zeta}: \mathrm{C}_{0}^{\infty}\left(\mathbb{R}^{2} \backslash \overline{B_{\gamma}}\right) \rightarrow \mathrm{L}^{2}\left(\mathbb{R}^{2} \backslash B_{\gamma}\right), \quad \psi \mapsto\left[-\Delta+\left[ \pm 2 m V+(1+1 / \zeta) V^{2}\right] /(1+\zeta)\right] \psi
$$

is bounded from below for all $\zeta \in(0,1)$. We denote its Friedrichs extension (see [12], Thm. X.23) by $\mathfrak{W}_{ \pm}^{\zeta}$. A form core for $\mathfrak{W}_{ \pm}^{\zeta}$ is given by $C_{0}^{\infty}\left(\mathbb{R}^{2} \backslash \overline{B_{\gamma}}\right)$.

In the following lemma, we reduce the problem to the study of negative eigenvalues of $\mathfrak{W}_{ \pm}^{\zeta}$ and $\mathfrak{V}_{ \pm}^{\zeta}$.

Lemma 13. There exist $c \in \mathbb{N}$ such that for all $\zeta \in(0,1)$ and $v \in \mathbb{R}^{-}:=(-\infty, 0)$ it holds that

$$
\sum_{+,-} \mathscr{N}_{(-\infty, v)}\left(\mathfrak{W}_{ \pm}^{\zeta}\right) \leq \mathscr{N}_{(-\infty, v)}\left(D^{2}-m^{2} \mathbb{I}\right) \leq c+\sum_{+,-} \mathscr{N}_{(-\infty, v)}\left(\mathfrak{V}_{ \pm}^{\zeta}\right)
$$

Proof. We first claim the existence of $\left\{\tilde{c}_{n}\right\}_{n=1}^{N} \in\left(\mathbb{R}^{+}\right)^{N}$ such that the inequality

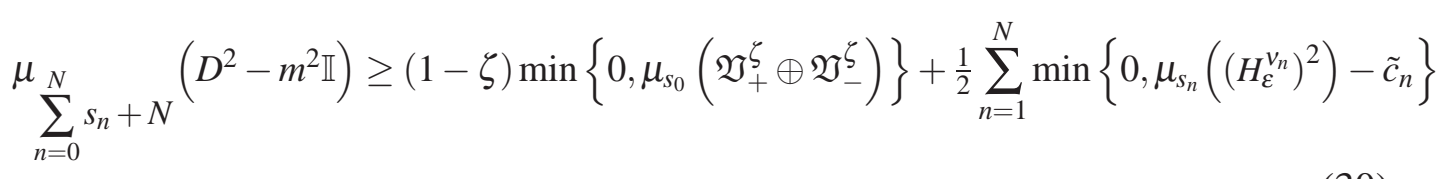

holds for all $\zeta \in(0,1)$ and $\left\{s_{n}\right\}_{n=0}^{N} \in \mathbb{N}^{N+1}$. 
One easily checks that the IMS localization formula for the quadratic form associated to $D^{2}-m^{2} \mathbb{I}$,

$$
\begin{aligned}
& \|D \cdot\|^{2}-m^{2}\|\cdot\|^{2}=\sum_{n=0}^{N} \mathfrak{v}\left[U_{n} \cdot\right], \\
& \mathfrak{v}: \mathscr{D}(D) \rightarrow \mathbb{R}, \quad \psi \mapsto\|D \psi\|^{2}-m^{2}\|\psi\|^{2}-\sum_{j=0}^{N}\left\|\left|\nabla U_{j}\right| \psi\right\|^{2},
\end{aligned}
$$

holds, and therefore Lem. 12 implies that

$$
\mu_{\sum_{n=0} s_{n}+N}\left(D^{2}-m^{2} \mathbb{I}\right) \geq \sup _{\substack{M \subset \mathrm{L}^{2}\left(\mathbb{R}^{2}, \mathbb{C}^{2}\right) \\ \operatorname{dim}(\operatorname{span}(M)) \leq \sum_{n=0}^{N} s_{n}-N-1}} \inf _{\substack{\psi \in \mathfrak{C}^{\prime} \cap M \perp \\\|\psi\|=1}} \sum_{j=0}^{N} \mathfrak{v}\left[U_{j} \psi\right] .
$$

The estimate

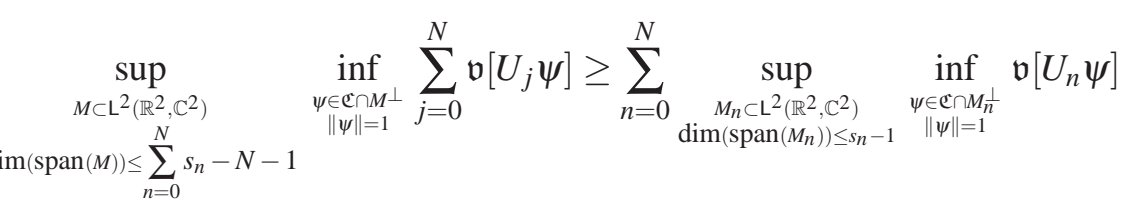

is trivial. Partially following Evans et al [4] (inequality (21)), we obtain

$$
\sup _{\substack{M_{n} \subset \mathrm{L}^{2}\left(\mathbb{R}^{2}, \mathbb{C}^{2}\right) \\ \operatorname{dim}\left(\operatorname{span}\left(M_{n}\right)\right) \leq s_{n}-1}} \inf _{\substack{\psi \in \mathfrak{C}^{n} \cap M_{n}^{\perp} \\\|\psi\|=1}} \mathfrak{v}\left[U_{n} \psi\right] \geq \sup _{\substack{M_{n} \subset \mathrm{L}^{2}\left(\mathbb{R}^{2}, \mathbb{C}^{2}\right) \\ \operatorname{dim}\left(\operatorname{span}\left(M_{n}\right)\right) \leq s_{n}-1}} \inf _{\substack{\psi \in \mathfrak{C} \cap\left(U_{n} \mathscr{A}_{n} M_{n}\right)^{\perp} \\\|\psi\|=1}} \mathfrak{v}\left[U_{n} \psi\right],
$$

where $\mathscr{A}_{n}: \mathrm{L}^{2}\left(\mathbb{R}^{2}, \mathbb{C}^{2}\right) \rightarrow \mathrm{L}^{2}\left(\mathbb{R}^{2}, \mathbb{C}^{2}\right), \psi \mapsto \psi\left(\cdot-x_{n}\right)$, where we set $x_{0}:=0$, and



The second step in (35) follows from the inclusion $U_{n}\left(U_{n} \mathscr{A}_{n} M_{n}\right)^{\perp} \subset \mathscr{A}_{n} M_{n}^{\perp}$. As $V \chi_{\text {supp }\left(U_{0}\right)} \in \mathrm{L}_{\infty}^{2}\left(\mathbb{R}^{2}\right)$ (see Hypothesis 5. (a) in Thm. 7), all $\psi \in \mathrm{C}_{0}^{\infty}\left(\mathbb{R}^{2}, \mathbb{C}^{2}\right)$ obey

$$
\begin{aligned}
\left|2 \Re\left\langle-i \boldsymbol{\sigma} \cdot \nabla \psi, V \chi_{\operatorname{supp}\left(U_{0}\right)} \psi\right\rangle\right| & \leq 2\|\nabla \psi\|\left\|V \chi_{\operatorname{supp}\left(U_{0}\right)} \psi\right\| \leq \\
& \leq\left\langle\psi,\left(-\zeta \Delta+V^{2} \chi_{\operatorname{supp}\left(U_{0}\right)} / \zeta\right) \psi\right\rangle
\end{aligned}
$$

(cf. [10], inequality (14)). If $\psi \equiv\left(\psi_{1}, \psi_{2}\right) \in U_{0} \mathfrak{C} \subset C_{0}^{\infty}\left(\operatorname{supp}\left(U_{0}\right), \mathbb{C}^{2}\right)$, then, with $\psi=\chi_{\operatorname{supp}\left(U_{0}\right)} \psi$, (36) implies that

$$
\begin{aligned}
\|D \psi\|^{2}-m^{2}\|\psi\|^{2} & \geq\left\langle\psi,\left((\zeta-1) \Delta+2 m V \chi_{\operatorname{supp}\left(U_{0}\right)} \sigma_{3}+(1-1 / \zeta) V^{2} \chi_{\operatorname{supp}\left(U_{0}\right)}\right) \psi\right\rangle \\
& =(1-\zeta)\left\langle\psi,\left(-\Delta+\left[2 m V \chi_{\operatorname{supp}\left(U_{0}\right)} \sigma_{3}+(1-1 / \zeta) V^{2} \chi_{\operatorname{supp}\left(U_{0}\right)}\right] /(1-\zeta)\right) \psi\right\rangle \\
& =(1-\zeta)\left\langle\psi_{1} \oplus \psi_{2},\left(\mathfrak{V}_{+}^{\zeta} \oplus \mathfrak{V}_{-}^{\zeta}\right) \psi_{1} \oplus \psi_{2}\right\rangle+\sum_{n=0}^{N}\left\|\left|\nabla U_{n}\right| \psi\right\|^{2}
\end{aligned}
$$

(cf. [10], inequality (16)), which is equivalent to

$$
\mathfrak{v}[\psi] \geq(1-\zeta)\left\langle\psi_{1} \oplus \psi_{2},\left(\mathfrak{V}_{+}^{\zeta} \oplus \mathfrak{V}_{-}^{\zeta}\right) \psi_{1} \oplus \psi_{2}\right\rangle .
$$


It follows from (35), (38) and $U_{0} \mathfrak{C} \subset \mathrm{C}_{0}^{\infty}\left(\mathbb{R}^{2}, \mathbb{C}^{2}\right)$ that

$$
\inf _{\substack{\psi \in \mathfrak{C} \cap\left(U_{0} M_{0} \perp \\\|\psi\|=1\right.}} \mathfrak{v}\left[U_{0} \psi\right] \geq(1-\zeta) \min \left\{0, \inf _{\substack{\psi_{1} \oplus \psi_{2} \in \mathcal{C}_{0}^{\infty}\left(\mathbb{R}^{2}, \mathbb{C}^{2}\right) \cap M_{0}^{\perp} \\\left\|\psi_{1} \oplus \psi_{2}\right\|=1}}\left\langle\psi_{1} \oplus \psi_{2},\left(\mathfrak{V}_{+}^{\zeta} \oplus \mathfrak{V}_{-}^{\zeta}\right) \psi_{1} \oplus \psi_{2}\right\rangle\right\} .
$$

Now, let $n \in\{1, \ldots, N\}$. Hypothesis 4 in Thm. 7 guarantees the existence of $c_{n}^{\prime} \in \mathbb{R}^{+}$satisfying

$$
\|D \psi\|^{2} \geq\left(\left\|\left(-i \sigma \cdot \nabla+v_{n}\left|\cdot-x_{n}\right|^{-1}\right) \psi\right\|^{2}-c_{n}^{\prime}\|\psi\|^{2}\right) / 2=\left(\left\|D_{\varepsilon}^{v_{n}} \mathscr{A}_{n}^{*} \psi\right\|^{2}-c_{n}^{\prime}\|\psi\|^{2}\right) / 2
$$

for all $\psi \in U_{n} \mathfrak{C} \subset C_{0}^{\infty}\left(\left(B_{\mathcal{\varepsilon}} \backslash\{0\}\right)+x_{n}\right)=\mathscr{A}_{n} \mathscr{D}\left(D_{\mathcal{\varepsilon}}^{v_{n}}\right)$. As $D_{\varepsilon}^{v_{n}} \subset H_{\mathcal{\varepsilon}}^{v_{n}}$, it follows that

$$
\inf _{\substack{\psi \in U_{n} \in \mathcal{C}^{n} \mathscr{A}_{n} M_{\bar{n}}^{-} \\\|\psi\|=1}} \mathfrak{v}[\psi] \geq \frac{1}{2} \inf _{\substack{\psi \in \mathscr{A}_{n}\left(\mathscr{D}\left(D_{\varepsilon}^{v_{n}}\right) \cap M_{\frac{1}{n}}\right) \\\|\psi\|=1}}\left(\left\|D_{\varepsilon}^{v_{n}} \mathscr{A}_{n}^{*} \psi\right\|^{2}-\tilde{c}_{n}\right) \geq \frac{1}{2} \inf _{\substack{\psi \in \mathscr{D}\left(H_{n}^{v_{n}}\right) \cap M_{\frac{1}{n}} \\\|\psi\|=1}}\left(\left\|H_{\varepsilon}^{v_{n}} \psi\right\|^{2}-\tilde{c}_{n}\right)
$$

holds for some $\tilde{c}_{n} \in \mathbb{R}^{+}$. Plugging (41) into (35), we obtain

$$
\inf _{\substack{\psi \in \mathfrak{C} \cap\left(U_{n} \mathscr{A}_{n} M_{n}\right)^{\perp} \\\|\psi\|=1}} \mathfrak{v}\left[U_{n} \psi\right] \geq \frac{1}{2} \min \left\{0, \inf _{\substack{\psi \in \mathscr{D}\left(H_{\varepsilon}^{v_{n}}\right) \cap M_{n}^{\perp} \\\|\psi\|=1}}\left(\left\|H_{\mathcal{E}}^{v_{n}} \psi\right\|^{2}-\tilde{c}_{n}\right)\right\} .
$$

Then, our preliminary claim (inequality (30) follows from (32)-(34), (39) and (42). With $s_{0}=\mathscr{N}_{(-\infty, \mathcal{v} /(1-\zeta))}\left(\mathfrak{V}_{+}^{\zeta} \oplus \mathfrak{V}_{-}^{\zeta}\right)+1$ and $s_{n}=\mathscr{N}_{\left(-\infty, \tilde{c}_{n}\right)}\left(\left(H_{\varepsilon}^{v_{n}}\right)^{2}\right)+1$, the right side - and thus the left side - of (30) is bounded from below by $v$ and hence

$$
\mathscr{N}_{(-\infty, v)}\left(D^{2}-m^{2} \mathbb{I}\right) \leq 2 N+\mathscr{N}_{(-\infty, v /(1-\zeta))}\left(\mathfrak{V}_{+}^{\zeta} \oplus \mathfrak{V}_{-}^{\zeta}\right)+\sum_{n=1}^{N} \mathscr{N}_{\left(-\infty, \tilde{c}_{n}\right)}\left(\left(H_{\varepsilon}^{v_{n}}\right)^{2}\right)
$$

holds. As the spectra of $\left\{H_{\varepsilon}^{v_{n}}\right\}_{n=1}^{N}$ are discrete (see Lem. 10), $\mathscr{N}_{\left(-\infty, \tilde{c}_{n}\right)}\left(\left(H_{\varepsilon}^{v_{n}}\right)^{2}\right)$ is finite for all $n \in\{1, \ldots, N\}$. Then, the upper bound in (29) follows from

$$
\mathscr{N}_{(-\infty, v /(1-\zeta))}\left(\mathfrak{V}_{+}^{\zeta} \oplus \mathfrak{V}_{-}^{\zeta}\right) \leq \mathscr{N}_{(-\infty, v)}\left(\mathfrak{V}_{+}^{\zeta} \oplus \mathfrak{V}_{-}^{\zeta}\right)=\sum_{+,-} \mathscr{N}_{(-\infty, v)}\left(\mathfrak{V}_{ \pm}^{\zeta}\right)
$$

As for the lower bound, by the Min-Max principle, the eigenvalues of $D^{2}-m^{2} \mathbb{I}$ are bounded from above by those of the Friedrichs extension of

$$
\mathrm{C}_{0}^{\infty}\left(\mathbb{R}^{2} \backslash \overline{B_{\gamma}}, \mathbb{C}^{2}\right) \rightarrow \mathrm{L}^{2}\left(\mathbb{R}^{2} \backslash B_{\gamma}, \mathbb{C}^{2}\right), \quad \psi \mapsto\left(D^{2}-m^{2} \mathbb{I}\right) \psi .
$$

As in (37), we estimate for all $\psi \equiv\left(\psi_{1}, \psi_{2}\right) \in \mathrm{C}_{0}^{\infty}\left(\mathbb{R}^{2} \backslash \overline{B_{\gamma}}, \mathbb{C}^{2}\right)$

$$
\begin{aligned}
\|D \psi\|^{2}-m^{2}\|\psi\|^{2} & \leq\left\langle\psi,\left(-(1+\zeta) \Delta+2 m V \sigma_{3}+(1+1 / \zeta) V^{2}\right) \psi\right\rangle \\
& =(1+\zeta)\left\langle\psi,\left(-\Delta+\left[2 m V \sigma_{3}+(1+1 / \zeta) V^{2}\right] /(1+\zeta)\right) \psi\right\rangle \\
& =(1+\zeta)\left\langle\psi_{1} \oplus \psi_{2},\left(\mathfrak{W}_{+}^{\zeta} \oplus \mathfrak{W}_{-}^{\zeta}\right) \psi_{1} \oplus \psi_{2}\right\rangle
\end{aligned}
$$

(cf. [10], inequality (15)). Then, the lower bound in (29) follows from

$$
\mathscr{N}_{(-\infty, v /(1+\zeta))}\left(\mathfrak{W}_{+}^{\zeta} \oplus \mathfrak{W}_{-}^{\zeta}\right) \geq \mathscr{N}_{(-\infty, v)}\left(\mathfrak{W}_{+}^{\zeta} \oplus \mathfrak{W}_{-}^{\zeta}\right)=\sum_{+,-} \mathscr{N}_{(-\infty, v)}\left(\mathfrak{W}_{ \pm}^{\zeta}\right) .
$$


At the expense of a bounded and compactly supported localization error, the negative eigenvalues of Schrödinger operators defined in $L^{2}\left(\mathbb{R}^{2}\right)$ with pure long range dipole potentials can be bounded from below by those of Schrödinger operators defined in $\mathrm{L}^{2}\left(\mathbb{R}^{2} \backslash \overline{B_{\gamma}}\right)$ with pure dipole potentials (see below). The latter accumulate exponentially fast at the bottom of the essential spectrum (see [10], Lem. 1). To decouple the interior from the exterior part, we make use of a further partition of unity $\left(\tilde{U}_{\text {int }}, \tilde{U}_{\text {ext }}\right) \in \mathrm{C}_{0}^{\infty}\left(B_{2 \gamma},[0,1]\right) \times \mathrm{C}^{\infty}\left(\mathbb{R}^{2} \backslash \overline{B_{\gamma}},[0,1]\right)$ with $\left(\tilde{U}_{\text {int }}\right)^{2}+\left(\tilde{U}_{\text {ext }}\right)^{2}=1$.

Lemma 14. Let $\mathfrak{c} \in \mathbb{R}^{2} \backslash\{0\}$ and $-\Delta_{\mathbb{R}^{2} \backslash \overline{B_{\gamma}}}^{\mathrm{D}}$ be the Dirichlet-Laplacian for $\mathbb{R}^{2} \backslash \overline{B_{\gamma}}$ (see [11], Section XIII.15). Then it holds for all $v \in \mathbb{R}^{-}$that

$$
\mathscr{N}_{(-\infty, v)}\left(-\Delta_{\mathbb{R}^{2}}+\chi_{\mathbb{R}^{2} \backslash B_{\gamma}}\langle\mathfrak{c}, \cdot\rangle_{\mathbb{R}^{2}} /|\cdot|^{3}+\mathscr{L}_{\gamma}^{\mathfrak{c}}\right) \leq \mathscr{N}_{(-\infty, v)}\left(-\Delta_{\mathbb{R}^{2} \backslash \overline{B_{\gamma}}}^{\mathrm{D}}+\langle\mathfrak{c}, \cdot\rangle_{\mathbb{R}^{2}} /|\cdot|^{3}\right),
$$

where $\mathscr{L}_{\gamma}^{\mathfrak{c}}:=\chi_{B_{2 \gamma} \backslash B_{\gamma}}\left|\langle\mathfrak{c}, \cdot\rangle_{\mathbb{R}^{2}}\right| /|\cdot|^{3}+\left|\nabla \tilde{U}_{\text {int }}\right|^{2}+\left|\nabla \tilde{U}_{\text {ext }}\right|^{2}$ is the localization error.

Proof. Let $M \subset \mathrm{L}^{2}\left(\mathbb{R}^{2}\right)$. As in (35), we estimate using the IMS localization formula for Schrödinger operators (see [2], Thm. 3.2)

$$
\begin{aligned}
& \inf _{\psi \in \mathrm{C}_{0}^{\infty}\left(\mathbb{R}^{2}\right) \cap\left(\tilde{U}_{\mathrm{ext}} M\right)^{\perp} \perp \|=1}\left\langle\psi,\left(-\Delta+\chi_{\mathbb{R}^{2} \backslash B_{\gamma}}\langle\mathfrak{c}, \cdot\rangle_{\mathbb{R}^{2}} /|\cdot|^{3}+\mathscr{L}_{\gamma}^{\mathfrak{c}}\right) \psi\right\rangle \\
& =\inf _{\psi \in \mathrm{C}_{0}^{\infty}\left(\mathbb{R}^{2}\right) \cap\left(\tilde{U}_{\mathrm{ext}} M\right)^{\perp}}\left[\left\langle\tilde{U}_{\mathrm{ext}} \psi,\left(-\Delta+\chi_{\mathbb{R}^{2} \backslash B_{\gamma}}\langle\mathfrak{c}, \cdot\rangle_{\mathbb{R}^{2}} /|\cdot|^{3}+\chi_{B_{2 \gamma} \backslash B_{\gamma}}\left|\langle\mathfrak{c}, \cdot\rangle_{\mathbb{R}^{2}}\right| /|\cdot|^{3}\right) \tilde{U}_{\mathrm{ext}} \psi\right\rangle+\right. \\
& \left.+\left\langle\tilde{U}_{\mathrm{int}} \psi,\left(-\Delta+\chi_{\mathbb{R}^{2} \backslash B_{\gamma}}\langle\mathfrak{c}, \cdot\rangle_{\mathbb{R}^{2}} /|\cdot|^{3}+\chi_{B_{2 \gamma} \backslash B_{\gamma}}\left|\langle\mathfrak{c}, \cdot\rangle_{\mathbb{R}^{2}}\right| /|\cdot|^{3}\right) \tilde{U}_{\mathrm{int}} \psi\right\rangle\right] \\
& \geq \inf _{\psi \in \mathrm{C}_{0}^{\infty}\left(\mathbb{R}^{2}\right) \cap\left(\tilde{U}_{\mathrm{ext}} M\right)^{\perp}}\left\langle\tilde{U}_{\mathrm{ext}} \psi,\left(-\Delta+\chi_{\mathbb{R}^{2} \backslash B_{\gamma}}\langle\mathfrak{c}, \cdot\rangle_{\mathbb{R}^{2}} /|\cdot|^{3}\right) \tilde{U}_{\mathrm{ext}} \psi\right\rangle \\
& \geq \inf _{\substack{\psi \in \mathrm{C}_{0}^{\infty}\left(\mathbb{R}^{2} \backslash \overline{B_{\gamma}}\right) \cap M^{\perp} \\
\|\psi\| \leq 1}}\left\langle\psi,\left(-\Delta+\langle\mathfrak{c}, \cdot\rangle_{\mathbb{R}^{2}} /|\cdot|^{3}\right) \psi\right\rangle .
\end{aligned}
$$

By an estimate similar to (34), we conclude that negative eigenvalues satisfy

$$
\mu_{s}\left(-\Delta_{\mathbb{R}^{2}}+\chi_{\mathbb{R}^{2} \backslash B_{\gamma}}\langle\mathfrak{c}, \cdot\rangle_{\mathbb{R}^{2}} /|\cdot|^{3}+\mathscr{L}_{\gamma}^{\mathfrak{c}}\right) \geq \mu_{s}\left(-\Delta_{\mathbb{R}^{2} \backslash \overline{B_{\gamma}}}^{\mathrm{D}}+\langle\mathfrak{c}, \cdot\rangle_{\mathbb{R}^{2}} /|\cdot|^{3}\right)
$$

which implies (48).

Next, following Rademacher and Siedentop [10], we decouple the pure dipole part from higher-order multipole moments, which - a posteriori - merely contribute with finitely many negative eigenvalues. For this purpose, we formulate the following statement.

Lemma 15. Suppose, $A_{1}, A_{2}$ and $A_{3}$ are lower semi-bounded self-adjoint operators in a Hilbert space with a common form core $K$ such that $\inf \sigma_{\mathrm{ess}}\left(A_{j}\right) \in \mathbb{R}_{0}^{+}$for $j=1,2,3$ is satisfied and $A_{1}=A_{2}+A_{3}$ holds in the form sense on $K$, i.e.,

$$
\left\langle\psi, A_{1} \psi\right\rangle=\left\langle\psi, A_{2} \psi\right\rangle+\left\langle\psi, A_{3} \psi\right\rangle \quad \forall \psi \in K .
$$

Then, it holds for all $\eta \in(0,1)$ and $v \in \mathbb{R}^{-}$that

$$
\mathscr{N}_{(-\infty, v)}\left(A_{1}\right) \leq \mathscr{N}_{(-\infty,(1-\eta) v)}\left(A_{2}\right)+\mathscr{N}_{(-\infty, \eta v)}\left(A_{3}\right) .
$$


Proof. The statement follows by mimicking the proof of Prop. 4. in [5].

Remark 16 . We obtain for all $\xi \in(0,1)$ and $v^{\prime} \in \mathbb{R}^{-}$the inequality

$$
\mathscr{N}_{\left(-\infty, v^{\prime}\right)}\left(A_{2}\right) \geq \mathscr{N}_{\left(-\infty,(1+\xi) v^{\prime}\right)}\left(A_{1}\right)-\mathscr{N}_{\left(-\infty, \xi v^{\prime}\right)}\left(A_{3}\right)
$$

when we insert $v=(1+\xi) v^{\prime}$ and $\eta=(1+\xi)^{-1} \xi$ into (52).

Let $\eta, \xi, \zeta \in(0,1)$. We decompose $\mathfrak{V}_{ \pm}^{\zeta}$ into $\mathfrak{V}_{ \pm}^{\zeta}=(1-\eta) \mathfrak{X}_{ \pm}^{\zeta, \eta}+\eta \mathfrak{T}_{ \pm}^{\zeta, \eta}$, where

$$
\mathfrak{X}_{ \pm}^{\zeta, \eta}:=-\Delta_{\mathbb{R}^{2}} \pm \chi_{\mathbb{R}^{2} \backslash B_{\gamma}} \frac{2 m}{(1-\zeta)(1-\eta)} \frac{\langle\mathfrak{d}, \cdot\rangle_{\mathbb{R}^{2}}}{|\cdot|^{3}}+\mathscr{L}_{\gamma}^{ \pm 2 m \mathfrak{d} /[(1-\zeta)(1-\eta)]}
$$

and

$$
\begin{aligned}
\mathfrak{T}_{ \pm}^{\zeta, \eta}:=-\Delta_{\mathbb{R}^{2}}+ & \left( \pm 2 m\left[V \chi_{\operatorname{supp}\left(U_{0}\right)}-\frac{\langle\mathfrak{d}, \cdot\rangle_{\mathbb{R}^{2}}}{|\cdot|^{3}} \chi_{\mathbb{R}^{2} \backslash B_{\gamma}}\right]+(1-1 / \zeta) V^{2} \chi_{\operatorname{supp}\left(U_{0}\right)}-\right. \\
& \left.-\sum_{n=0}^{N}\left|\nabla U_{n}\right|^{2}-(1-\eta)(1-\zeta) \mathscr{L}_{\gamma}^{ \pm 2 m \mathfrak{d} /[(1-\eta)(1-\zeta)]}\right) /[(1-\zeta) \eta]
\end{aligned}
$$

Since $V \chi_{\text {supp }\left(U_{0}\right)}$ and $V^{2} \chi_{\text {supp }}\left(U_{0}\right)$ are $\left(-\Delta_{\mathbb{R}^{2}}\right)$-compact (see above), $\mathfrak{T}_{ \pm}^{\zeta, \eta}$ is selfadjoint and bounded from below.

Using Lem. 15 and then Lem.14, we obtain for all $v \in \mathbb{R}^{-}$that

$$
\begin{aligned}
\mathscr{N}_{(-\infty, v)}\left(\mathfrak{V}_{ \pm}^{\zeta}\right) & \leq \mathscr{N}_{(-\infty, v)}\left(\mathfrak{X}_{ \pm}^{\zeta, \eta}\right)+\mathscr{N}_{(-\infty, v)}\left(\mathfrak{T}_{ \pm}^{\zeta, \eta}\right) \\
& \leq \mathscr{N}_{(-\infty, v)}\left(-\Delta_{\mathbb{R}^{2} \backslash \overline{B_{\gamma}}}^{\mathrm{D}} \pm \frac{2 m}{(1-\zeta)(1-\eta)} \frac{\langle\mathfrak{d},\rangle_{\mathbb{R}^{2}}}{|\cdot|^{3}}\right)+\mathscr{N}_{\mathbb{R}^{-}}\left(\mathfrak{T}_{ \pm}^{\zeta, \eta}\right) .
\end{aligned}
$$

We decompose $\mathfrak{W}_{ \pm}^{\zeta}$ in a similar way. Let $\mathfrak{Z}_{ \pm}^{\zeta, \xi}$ be the Friedrichs extension of

$$
\mathrm{C}_{0}^{\infty}\left(\mathbb{R}^{2} \backslash \overline{B_{\gamma}}\right) \rightarrow \mathrm{L}^{2}\left(\mathbb{R}^{2} \backslash B_{\gamma}\right), \quad \psi \mapsto-\Delta \psi+\left[\mp 2 m\left[V-\frac{\langle\mathfrak{d}, \cdot\rangle_{\mathbb{R}^{2}}}{|\cdot|^{3}}\right]-(1+1 / \zeta) V^{2}\right] \psi /[(1+\zeta) \xi],
$$

which is bounded from below, since $\chi_{\mathbb{R}^{2} \backslash B_{\gamma}} V$ and $\chi_{\mathbb{R}^{2} \backslash B_{\gamma}} V^{2}$ are $\left(-\Delta_{\mathbb{R}^{2}}\right)$-compact (cf. above considerations).

Then, since

$$
(1+\xi)\left(-\Delta_{\mathbb{R}^{2} \backslash \overline{B \gamma}}^{\mathrm{D}} \pm \frac{2 m}{(1+\zeta)(1+\xi)} \frac{\langle\mathcal{D}, \cdot\rangle_{\mathbb{R}^{2}}}{|\cdot|^{3}}\right)=\mathfrak{W}_{ \pm}^{\zeta}+\xi \mathfrak{Z}_{ \pm}^{\zeta, \xi}
$$

holds in the form sense on the common form core $C_{0}^{\infty}\left(\mathbb{R}^{2} \backslash \overline{B_{\gamma}}\right)$, we obtain

$$
\begin{aligned}
\mathscr{N}_{(-\infty, v)}\left(\mathfrak{W}_{ \pm}^{\zeta}\right) & \geq \mathscr{N}_{(-\infty, v)}\left(-\Delta_{\mathbb{R}^{2} \backslash \overline{B_{\gamma}}}^{\mathrm{D}} \pm \frac{2 m}{(1+\zeta)(1+\xi)} \frac{\langle\mathfrak{d},\rangle_{\mathbb{R}^{2}}}{|\cdot|^{3}}\right)-\mathscr{N}_{(-\infty, v)}\left(\mathfrak{Z}_{ \pm}^{\zeta, \xi}\right) \\
& \geq \mathscr{N}_{(-\infty, v)}\left(-\Delta_{\mathbb{R}^{2} \backslash \bar{B}_{\gamma}}^{\mathrm{D}} \pm \frac{2 m}{(1+\zeta)(1+\eta)} \frac{\langle\mathfrak{d},\rangle_{\mathbb{R}^{2}}}{|\cdot|^{3}}\right)-\mathscr{N}_{\mathbb{R}^{-}}\left(\mathfrak{Z}_{ \pm}^{\zeta, \xi}\right) .
\end{aligned}
$$

for all $v \in \mathbb{R}^{-}$by using (53).

As mentioned above, we now show that the higher-order multipole moments merely contribute with finitely many negative eigenvalues. 
Lemma 17. Let $\zeta, \eta, \xi \in(0,1)$. Then, $\mathscr{N}_{\mathbb{R}^{-}}\left(\mathfrak{T}_{ \pm}^{\zeta, \eta}\right)$ and $\mathscr{N}_{\mathbb{R}^{-}}\left(\mathfrak{Z}_{ \pm}^{\zeta, \xi}\right)$ are finite.

Proof. It follows from Hypothesis 5.(a) in Thm. 7 that $V \chi_{\operatorname{supp}\left(U_{0}\right)}-\frac{\langle\hat{d}, \cdot\rangle_{\mathbb{R}^{2}}}{|\cdot|^{3}} \chi_{\mathbb{R}^{2}} \backslash B_{\gamma}$ and $V^{2} \chi_{\operatorname{supp}\left(U_{0}\right)}$ - and thus also the potential of $\mathfrak{T}_{ \pm}^{\zeta, \eta}$ - lie in $\mathrm{L}^{1}\left(\mathbb{R}^{2} ; \log (2+|x|) \mathrm{d} x\right)$. Accordingly, Hypothesis 5.(b) in Thm. 7implies that their spherical rearrangements are contained in $\mathrm{L}^{1}\left(\mathbb{R}^{+} ; \log _{+}\left(r^{-1}\right) \mathrm{d} r\right)$. Then, the finiteness of $\mathscr{N}_{\mathbb{R}^{-}}\left(\mathfrak{T}_{ \pm}^{\zeta, \eta}\right)$ follows from Thm. 4.3 in [15]. The same applies to the zero extension of the potential in (57) to $\mathbb{R}^{2}$. Hence - by the inclusion of form cores $C_{0}^{\infty}\left(\mathbb{R}^{2} \backslash \overline{B_{\gamma}}\right) \subset C_{0}^{\infty}\left(\mathbb{R}^{2}\right)$ Thm. 4.3 in [15] also implies that $\mathscr{N}_{\mathbb{R}^{-}}\left(\mathfrak{Z}_{ \pm}^{\zeta, \xi}\right)$ is finite.

We are now prepared for the proof of Thm. 77:

Proof. Let $\zeta, \eta, \xi \in(0,1)$. Using Lem. 13 and 17 and inequalities (56) and (59), we estimate

$$
\begin{aligned}
& \limsup _{E \nearrow m} \frac{\mathscr{N}_{\left(-\infty, E^{2}-m^{2}\right)}\left(D^{2}-m^{2} \mathbb{I}\right)}{|\log (m-E)|} \leq \\
& \leq \sum_{+,-\lim _{\nearrow} m} \frac{\mathscr{N}_{\left(-\infty, E^{2}-m^{2}\right)}\left(-\Delta_{\mathbb{R}^{2} \backslash \overline{B_{\gamma}}}^{\mathrm{D}} \pm \frac{2 m}{(1-\zeta)(1-\eta)} \frac{\langle\mathfrak{d}, \cdot\rangle_{\mathbb{R}^{2}}}{|\cdot|^{3}}\right)}{\left|\log \left(m^{2}-E^{2}\right)\right|} \overbrace{\left|\frac{\log \left(m^{2}-E^{2}\right)}{\log (m-E)}\right|}^{\rightarrow 1}
\end{aligned}
$$

and

$$
\begin{aligned}
& \liminf _{E \nearrow m} \frac{\mathscr{N}_{\left(-\infty, E^{2}-m^{2}\right)}\left(D^{2}-m^{2} \mathbb{I}\right)}{|\log (m-E)|} \geq \\
& \geq \sum_{+,-} \liminf _{E \nearrow m} \frac{\mathscr{N}_{\left(-\infty, E^{2}-m^{2}\right)}\left(-\Delta_{\mathbb{R}^{2} \backslash \overline{B_{\gamma}}}^{\mathrm{D}} \pm \frac{2 m}{(1+\zeta)(1+\xi)} \frac{\langle\mathfrak{d}, \cdot\rangle_{\mathbb{R}^{2}}}{|\cdot|^{3}}\right)}{\left|\log \left(m^{2}-E^{2}\right)\right|} \overbrace{\left|\frac{\log \left(m^{2}-E^{2}\right)}{\log (m-E)}\right|}^{\rightarrow 1} .
\end{aligned}
$$

Due to the continuity of $\operatorname{tr}\left(\sqrt{\left(M_{(\cdot)}\right)_{-}}\right)$(see [10]), the desired result follows from Lem. 1 in [10] in the limits $\zeta, \eta \rightarrow 0$ and $\zeta, \xi \rightarrow 0$, respectively.

\section{ACKNOWLEDGEMENTS}

I thank Heinz Siedentop and Sergey Morozov for many useful discussions.

\section{REFERENCES}

[1] J.-C. Cuenin and H. Siedentop. "Dipoles in graphene have infinitely many bound states". Journal of Mathematical Physics 55 (2014), p. 122304.

[2] H. L. Cycon, R. G. Froese, W. Kirsch, and B. Simon. ”Schrödinger Operators: with Application to Quantum Mechanics and Global Geometry". Texts and Monographics in Physics. Berlin: Springer-Verlag, 1987.

[3] A. De Martino, D. Klöpfer, D. Matrasulov, and R. Egger. "Electric-DipoleInduced Universality for Dirac Fermions in Graphene". Physical Review Letters 112 (2014), p. 186603. 
[4] W. D. Evans, R. T. Lewis, H. Siedentop, and J. P. Solovej. "Counting eigenvalues using coherent states with an application to Dirac and Schrödinger operators in the semi-classical limit". Arkiv för Matematik 34 (1996), pp. 265-283.

[5] W. Kirsch and B. Simon. "Corrections to the Classical Behavior of the Number of Bound States of Schrödinger Operators". Annals of Physics 183 (1988), pp. 122-130.

[6] N. W. McLachlan. Theory and Application of Mathieu Functions. Oxford, at the Clarenden Press, 1947.

[7] S. Morozov and D. Müller. "Lieb-Thirring and Cwickel-Lieb-Rozenblum inequalities for perturbed graphene wih a Coulomb impurity". Preprint arXiv: 1603.01485. 2016.

[8] S. Morozov and D. Müller. "On the virtual levels of positively projected massless Coulomb-Dirac operators". Preprint arXiv: 1607.08902. 2016.

[9] D. Müller. "Minimax principles, Hardy-Dirac inequalities and operator cores for two and three dimensional Coulomb-Dirac operators". Documenta Mathematica 21 (2016), pp. 1151-1169.

[10] S. Rademacher and H. Siedentop. "Accumulation rate of bound states of dipoles in graphene”. Journal of Mathematical Physics 57 (2016), p. 042105.

[11] M. Reed and B. Simon. "Analysis of Operators". Methods of Modern Mathematical Physics. Vol. 4. San Diego: Academic Press, 1978.

[12] M. Reed and B. Simon. "Fourier Analysis, Self-Adjointness". Methods of Modern Mathematical Physics. Vol. 2. San Diego: Academic Press, 1975.

[13] K. Schmüdgen. "Unbounded Self-adjoint Operators on Hilbert Space”. Graduate Texts in Mathematics. Dordrecht: Springer, 2012.

[14] E. Seiler and B. Simon. "Bounds in the Yukawa 2 Quantum Field Theory: Upper bound on the pressure, Hamiltonian bound and linear lower bound." Communications in Mathematical Physics 45 (1975), pp. 99-114.

[15] E. Shargorodsky. "On negative eigenvalues of two-dimensional Schrödinger operators". Proceedings of the London Mathematical Society 108 (2014), pp. 441-483.

[16] B. Thaller "The Dirac Equation". Texts and Monographics in Physics. Berlin: Springer-Verlag, 1992.

[17] M. A. H. Vozmediano, M. I. Katnelson, and F. Guinea. "Gauge fields in graphene". Physics Reports 496 (2010), pp. 109-148.

[18] J. Weidmann. Lineare Operatoren in Hilberträumen. Teil II: Anwendungen. B. G. Teubner, 2003.

[19] J. Weidmann "Oszillationsmethoden für Systeme gewöhnlicher Differentialgleichungen". Mathematische Zeitschrift 119 (1971), pp. 349-373. 